\title{
On a Recurrence Arising in Graph Compression*
}

\author{
Yongwook Choi \\ J. Craig Venter Institute \\ Rockville, MD, USA 20850 \\ ychoi@jcvi.org
}

\author{
Charles Knessl
}

Dept. Math. Stat. \& Comp. Sci.

University of Illinois at Chicago

Chicago, IL, USA 60607

knessl@uic.edu

\author{
Wojciech Szpankowski ${ }^{\dagger}$ \\ Dept. Computer Science \\ Purdue University \\ West Lafayette, IN, USA 47907 \\ spa@cs.purdue.edu
}

Submitted: November 16, 2011; Accepted: July 11, 2012; Published: Aug 9, 2012

Mathematics Subject Classification: 05C05, 68W40

\begin{abstract}
In a recently proposed graphical compression algorithm by Choi and Szpankowski (2012), the following tree arose in the course of the analysis. The root contains $n$ balls that are consequently distributed between two subtrees according to a simple rule: In each step, all balls independently move down to the left subtree (say with probability $p$ ) or the right subtree (with probability $1-p$ ). A new node is created as long as there is at least one ball in that node. Furthermore, a nonnegative integer $d$ is given, and at level $d$ or greater one ball is removed from the leftmost node before the balls move down to the next level. These steps are repeated until all balls are removed (i.e., after $n+d$ steps). Observe that when $d=\infty$ the above tree can be modeled as a trie that stores $n$ independent sequences generated by a binary memoryless source with parameter $p$. Therefore, we coin the name $(n, d)$-tries for the tree just described, and to which we often refer simply as $d$-tries. We study here in detail the path length, and show how much the path length of such a $d$-trie differs from that of regular tries. We use methods of analytic algorithmics, from Mellin transforms to analytic poissonization.
\end{abstract}

*This work was supported in part by the NSF Science and Technology Center for Science of Information Grant CCF-0939370, NSF Grant CCF-0830140, AFOSR Grant FA8655-11-1-3076, NSA Grants H9823011-1-0141 and H98230-11-1-0184, and the MNSW grant N206 369739.

${ }^{\dagger}$ Also, Visiting Professor at ETI, Gdansk University of Technology, Poland. 


\section{Introduction}

In [1] an algorithm was described to compress the structure of a graph. The main idea behind the algorithm is quite simple: First, a vertex of a graph, say $v_{1}$, is selected and the number of neighbors of $v_{1}$ is stored in a binary string. Then the remaining $n-1$ vertices are partitioned into two sets: the neighbors of $v_{1}$ and the non-neighbors of $v_{1}$. This process continues by selecting randomly a vertex, say $v_{2}$, from the neighbors of $v_{1}$ and storing two numbers: the number of neighbors of $v_{2}$ among each of the above two sets. Then the remaining $n-2$ vertices are partitioned into four sets: the neighbors of both $v_{1}$ and $v_{2}$, the neighbors of $v_{1}$ that are non-neighbors of $v_{2}$, the non-neighbors of $v_{1}$ that are neighbors of $v_{2}$, and the non-neighbors of both $v_{1}$ and $v_{2}$. This procedure continues until all vertices are processed.

In the Erdős-Rényi model, a random graph has any pair of vertices connected by an edge with probability $p$. It is proved in [1] that for large $n$ our algorithm optimally compresses any graph generated by the Erdős-Rényi model (and, in fact, it works well in practice even for graphs not generated by the Erdős-Rényi model). To establish this asymptotic optimality result, an interesting tree was used in the construction, that we describe next.

The root of such a tree contains $n$ balls (vertices of the underlying graph) that are consequently distributed between two subtrees according to a simple rule: In each step, all balls independently move down to the left subtree (say with probability $p$ ) or the right subtree (with probability $q=1-p$ ), and a new node is created as long as there is at least one ball in that node. Finally, a non-negative integer $d$ is given so that at level $d$ or greater one ball is removed from the leftmost node before the balls move down to the next level. These steps are repeated until all balls are removed (i.e., after $n+d$ steps). Of interest are such tree parameters as the depth, path length (sum of all depths), size, and so forth. This is illustrated in Figure 1 in which the deleted ball is shown next to the node from where it was removed.

The tree just described falls between two digital trees, namely tries and digital search trees. In fact, when $d=\infty$ the tree can be modeled as a trie that stores $n$ independent sequences generated by a binary memoryless source with parameter $p$. Hence, we coin the term $(n, d)$-trie (or simply $d$-trie) for the tree just described. In [1] lower and upper bounds were proved for parameters of interest, by using known results for tries and digital search trees $[3,19]$. In this paper, we establish precise asymptotic results. In particular, we show by how much the path length of a $d$-trie differs from the path length of the corresponding regular trie.

Many parameters of a $d$-trie can be described by the following two dimensional recurrence

$$
a(n, d)=f(n)+\sum_{k=0}^{n}\left(\begin{array}{l}
n \\
k
\end{array}\right) p^{k} q^{n-k}[a(k, d-1)+a(n-k, k+d-1)], \quad d \geqslant 1,
$$

THE ELECTRONiC JOURNAL OF COMBINATORICS 19(3) (2012), \#P15 


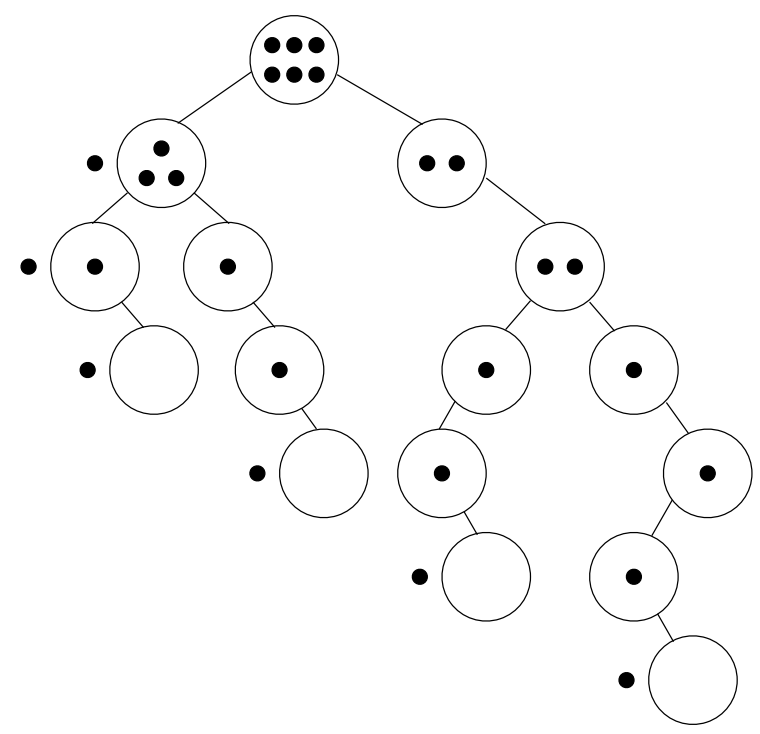

Figure 1: A $(6,1)$-trie with six balls and $d=1$, in which the deleted ball is shown next to the node where it was removed.

with $q=1-p$, and the boundary equation

$$
a(n+1,0)=f(n)+\sum_{k=0}^{n}\left(\begin{array}{l}
n \\
k
\end{array}\right) p^{k} q^{n-k}[a(k, 0)+a(n-k, k)],
$$

for a known additive term $f(n)$. For example, when $f(n)=n$, then $a(n, d)$ represents the path length. Recurrence (2) is equivalent to the following boundary condition

$$
a(n, 1)=a(n+1,0) .
$$

For $d=\infty$ recurrence (1) becomes a traditional recurrence arising in the analysis of tries [19] whose solutions (exact and asymptotic) are well known. Thus, it is natural to study the difference $\tilde{a}(n, d):=a(n, \infty)-a(n, d)$, and that is our objective. In passing, we should point out that recurrence (2) resembles the one used to analyze another digital search tree, known as a digital search tree. In this paper we prove, however, that a $(n, d)$-trie more closely resembles a trie, rather than a digital search tree.

Our main interest lies in solving recurrence (1) for $d=O(1)$. In fact, for graph compression we only need $d=0$, and we focus on this case. We shall show that the term in (1) involving the sum over $a(n-k, k+d-1)$ becomes exponentially small for $n$ large and $d$ fixed. Then we shall approximate the recurrence for the excess quantity $\tilde{a}(n, d)$ by

$$
\tilde{a}(n, d)=\sum_{k=0}^{n}\left(\begin{array}{l}
n \\
k
\end{array}\right) p^{k} q^{n-k} \tilde{a}(k, d-1)
$$

with an appropriate initial condition. The above we can solve asymptotically using Mellin transform technique and depoissonization. In particular, for $f(n)=n$ (that is, for the 
path length in a $d$-trie) we prove that the excess quantity $\tilde{a}(n, d)$ becomes asymptotically, as $n \rightarrow \infty$ and $d=O(1)$,

$$
\frac{1}{2 h \log (1 / p)} \log ^{2} n-\frac{d}{h} \log n+\left[\frac{1}{2 h}-\frac{1}{h \log p}\left(\gamma+1+\frac{h_{2}}{2 h}+\Psi\left(\log _{p} n\right)\right)\right] \log n
$$

where $\Psi(\cdot)$ is the periodic function when $\log p / \log (1-p)$ is rational, and $h$ is the entropy rate.

Digital trees such as tries and digital search trees have been intensively studied for the last thirty years $[2,3,5,7,11,12,13,16,17,18,19]$. However, our two-dimensional recurrence seems to be new and harder to analyze. It somewhat resembles the profile recurrences for digital trees, which were studied for tries in [15] and digital search trees in [4], and which are known to also be challenging.

The paper is organized as follows. In the Section 2 we precisely formulate our problem and analyze it for $f(n)=n$. Proofs are presented in Section 3, where we also discuss some asymptotics for $d \rightarrow \infty$.

\section{Problem Statement}

In this section, we first formulate some recurrences describing $(n, d)$-tries, then summarize our main results, discuss some extensions, and present numerical results.

\subsection{Main Results}

Let us consider a $(n, d)$-trie with $n$ balls and parameter $d \geqslant 0$. First, we analyze the average path length $b(n, d)$. It satisfies the following recurrence equations

$$
b(n+1,0)=n+\sum_{k=0}^{n}\left(\begin{array}{l}
n \\
k
\end{array}\right) p^{k} q^{n-k}[b(k, 0)+b(n-k, k)], \text { for } n \geqslant 2,
$$

and

$$
b(n, d)=n+\sum_{k=0}^{n}\left(\begin{array}{l}
n \\
k
\end{array}\right) p^{k} q^{n-k}[b(k, d-1)+b(n-k, k+d-1)], \text { for } n \geqslant 2, d \geqslant 1 .
$$

Recurrence (3) follows from the fact that starting with $n+1$ balls in the root node, and removing one ball, we are left with $n$ balls passing through the root node. The root contributes $n$ since each time a ball moves down it adds 1 to the path length. Those $n$ balls move down to the left or the right subtrees. Let us assume $k$ balls move down to the left subtree (the other $n-k$ balls must move down to the right subtree); this occurs with probability $\left(\begin{array}{l}n \\ k\end{array}\right) p^{k} q^{n-k}$. At level one, one ball is removed from those $k$ balls in the root of the left subtree. This contributes $b(k, 0)$. There will be no removal from $n-k$ balls in the right subtree until all $k$ balls in the left subtree are removed. This contributes $b(n-k, k)$. Similarly, for $d>0$ we arrive at recurrence (4). 
Here $0<p<1$ and $q=1-p$, and we also use the boundary conditions

$$
b(0, d)=b(1, d)=0, \quad d \geqslant 0 ; \quad b(2,0)=0 .
$$

By setting $d=1$ in (4) and comparing the result to (3) we can replace (3) by the simpler boundary condition

$$
b(n, 1)=b(n+1,0), \text { for } n \geqslant 0 .
$$

We are primarily interested in estimating $b(n, 0)$ for large $n$.

If we let $d \rightarrow \infty$ in (4) and assume that $b(n, d)$ tends to a limit $b(n, \infty)$, then (4) becomes

$$
b(n, \infty)=n+\sum_{k=0}^{n}\left(\begin{array}{l}
n \\
k
\end{array}\right) p^{k} q^{n-k}[b(k, \infty)+b(n-k, \infty)],
$$

with $b(0, \infty)=b(1, \infty)=0$. This is the same as the recurrence for the mean path length in a trie, which was analyzed, for example, in $[12,19]$. One form of the solution is given by the alternating sum

$$
b(n, \infty)=\sum_{\ell=2}^{n}(-1)^{\ell}\left(\begin{array}{l}
n \\
\ell
\end{array}\right) \frac{\ell}{1-p^{\ell}-q^{\ell}},
$$

and an alternative form is given by the generating function

$$
\sum_{n=0}^{\infty} b(n, \infty) \frac{z^{n}}{n !} e^{-z}=\frac{1}{2 \pi i} \int_{B r} z^{-s} \frac{\Gamma(s+1)}{1-p^{-s}-q^{-s}} d s,
$$

where $\Gamma(\cdot)$ is the Gamma function and $B r$ is a vertical Bromwich contour on which $-2<\Re(s)<-1$. The integral certainly converges for $z$ real and positive.

The asymptotic expansion of $b(n, \infty)$ in (8) or (9) as $n \rightarrow \infty$ may be obtained by a combination of singularity analysis and depoissonization arguments (see $[7,8,19]$ ) and we obtain

$$
b(n, \infty)=\frac{1}{h} n \log n+\frac{1}{h}\left[\gamma+\frac{h_{2}}{2 h}+\Phi\left(\log _{p} n\right)\right] n+o(n),
$$

where $h=-p \log p-q \log q, h_{2}=p \log ^{2} p+q \log ^{2} q, \gamma$ is the Euler constant, and $\Phi(x)$ is the periodic function

$$
\Phi(x)=\sum_{k=-\infty, k \neq 0}^{\infty} \Gamma\left(-\frac{2 k \pi i r}{\log p}\right) e^{2 k \pi r i x},
$$

provided that $\log p / \log q=r / s$ is rational, with $r$ and $s$ being integers with $\operatorname{gcd}(r, s)=1$. If $\log p / \log q$ is irrational, then the term with $\Phi$ is absent from the $O(n)$ term of $(10)$.

Our analysis requires a two-term asymptotic estimate of the difference $b(n+1, \infty)-$ $b(n, \infty)$, whose generating function may be represented, similarly to (10), as the inverse Mellin transform

$$
\sum_{n \geqslant 0}[b(n+1, \infty)-b(n, \infty)] \frac{z^{n}}{n !} e^{-z}=\frac{1}{2 \pi i} \int_{B r} \frac{-s \Gamma(s+1)}{1-p^{-s}-q^{-s}} z^{-s-1} d s
$$


This integral has a double pole at $s=-1$, and we can obtain a two-term approximation for $z \rightarrow \infty$, which by depoissonization becomes

$$
b(n+1, \infty)-b(n, \infty)=\frac{1}{h} \log n+\frac{1}{h}\left(\gamma+1+\frac{h_{2}}{2 h}\right)+\frac{1}{h} \Psi\left(\log _{p} n\right)+o(1)
$$

where $\Psi(\cdot)$ is the periodic function in Theorem 1 , which again appears only for rational $\log p / \log q$.

The $o(1)$ term in (12), just as the term $o(n)$ in (10), is difficult to characterize explicitly, but our analysis requires only the first two terms of the asymptotic estimate in (12). Note that to obtain the leading term $O\left(\log ^{2} n\right)$ in Theorem 1, we only need the leading term in (12).

Next we set

$$
b(n, d)=b(n, \infty)-\tilde{b}(n, d)
$$

so that $\tilde{b}(n, d)=b(n, \infty)-b(n, d)$ measures how the path lengths in the $d$-trie differ from those in a trie. From (4) and (7), we then obtain

$$
\tilde{b}(n, d)=\sum_{k=0}^{n}\left(\begin{array}{l}
n \\
k
\end{array}\right) p^{k} q^{n-k}[\tilde{b}(k, d-1)+\tilde{b}(n-k, k+d-1)], \text { for } n \geqslant 2, d \geqslant 1,
$$

which unlike (4) is a homogeneous recurrence. Then from (6) and (13) we have the boundary condition

$$
\tilde{b}(n+1,0)-\tilde{b}(n, 1)=b(n+1, \infty)-b(n, \infty) .
$$

From $(5)$ and $(7)$ we also have $\tilde{b}(0, d)=\tilde{b}(1, d)=0$ for $d \geqslant 0$.

We further define $b_{*}(n, d)$ to be the solution of

$$
b_{*}(n, d)=\sum_{k=0}^{n}\left(\begin{array}{l}
n \\
k
\end{array}\right) p^{k} q^{n-k} b_{*}(k, d-1), \text { for } n \geqslant 2, d \geqslant 1,
$$

and

$$
b_{*}(n+1,0)-b_{*}(n, 1)=b(n+1, \infty)-b(n, \infty) .
$$

Note that (16) differs from (14) in that the former neglects the term involving $\tilde{b}(n-$ $k, k+d-1)$. We will show that this term in (14) is asymptotically negligible for $n \rightarrow \infty$ with $d=O(1)$, so that $\tilde{b}(n, d) \sim b_{*}(n, d)$. The recurrence (16) is much easier to solve by transform methods $[7,19]$ than is $(14)$.

We summarize our main result below. In Section 3 we establish Theorem 1 along with some other exact and asymptotic results for (3)-(6) and (14)-(17).

Theorem 1 For $n \rightarrow \infty$ and $d=O(1)$ the difference $b(n, \infty)-b(n, d)=\tilde{b}(n, d)$, which is the difference in path length between the present tree and a standard trie, is of order $O\left(\log ^{2} n\right)$ for $n \rightarrow \infty$. More precisely

$\tilde{b}(n, d)=\frac{\log ^{2} n}{2 h \log (1 / p)}-\frac{d \log n}{h}+\left[\frac{1}{2 h}-\frac{1}{h \log p}\left(\gamma+1+\frac{h_{2}}{2 h}+\Psi\left(\log _{p} n\right)\right)\right] \log n+O(1)$, 
where $\Psi(\cdot)$ is the periodic function

$$
\Psi(x)=\sum_{k=-\infty, k \neq 0}^{\infty}\left[1+\frac{2 k \pi i r}{\log p}\right] \Gamma\left(-\frac{2 k \pi i r}{\log p}\right) e^{2 k \pi i r x}
$$

and $\log p / \log q=r / t$ is rational. If $\log p / \log q$ is irrational, the term involving $\Psi$ is absent.

We see that $b(n, \infty)-b(n, d)=O\left(\log ^{2} n\right)$, which shows that the $(n, d)$-tries studied in [1] are in some sense more similar to tries than to digital search trees (DST). In [1], it was shown that $b(n, 0)$ was bounded above by average path lengths in tries and below by average path lengths in DST's. It was also conjectured that $b(n, \infty)-b(n, d)$ is $O(n)$, but our result shows that this difference is in fact much smaller.

\section{$2.2 \quad$ Related Recurrence Equations}

The method presented in the next section, allow us to analyze a class of recurrences of the type (3) with inhomogeneous terms other than $n$. For example, suppose we define $a(n, d)$ by

$$
a(n, d)=f(n)+\sum_{k=0}^{n}\left(\begin{array}{l}
n \\
k
\end{array}\right) p^{k} q^{n-k}[a(k, d-1)+a(n-k, k+d-1)]
$$

where $f(n)$ is a given sequence. The boundary condition is again of the type (3), or equivalently,

$$
a(n, 1)=a(n+1,0),
$$

and we have $a(0, d)=a(1, d)=0$. Also, let $a(n, \infty)$ satisfy (18) with the second argument of $a(\cdot, \cdot)$ replaced by infinity. This recurrence can be solved by generating functions and Mellin transforms, and we can then establish that $a(n, \infty)-a(n, d)=\tilde{a}(n, d)$, will satisfy

$$
\tilde{a}(n, d)=\sum_{k=0}^{n}\left(\begin{array}{l}
n \\
k
\end{array}\right) p^{k} q^{n-k}[\tilde{a}(k, d-1)+\tilde{a}(n-k, k+d-1)]
$$

and

$$
\tilde{a}(n+1,0)-\tilde{a}(n, 1)=a(n+1, \infty)-a(n, \infty) .
$$

The asymptotic behavior of $\tilde{a}(n, d)$ for $d=O(1)$ and $n \rightarrow \infty$ can be obtained in a manner completely analogous to the case $f(n)=n$, discussed in the next section.

For example, the case

$$
f(n)=\lceil\log (n+1)\rceil
$$

arose in analyzing the compression algorithm in [1]. In [1] it was shown that $a(n, \infty)$ has the asymptotic form

$$
a(n, \infty)=\frac{n}{h} A_{*}(-1)+o(n), \quad n \rightarrow \infty
$$




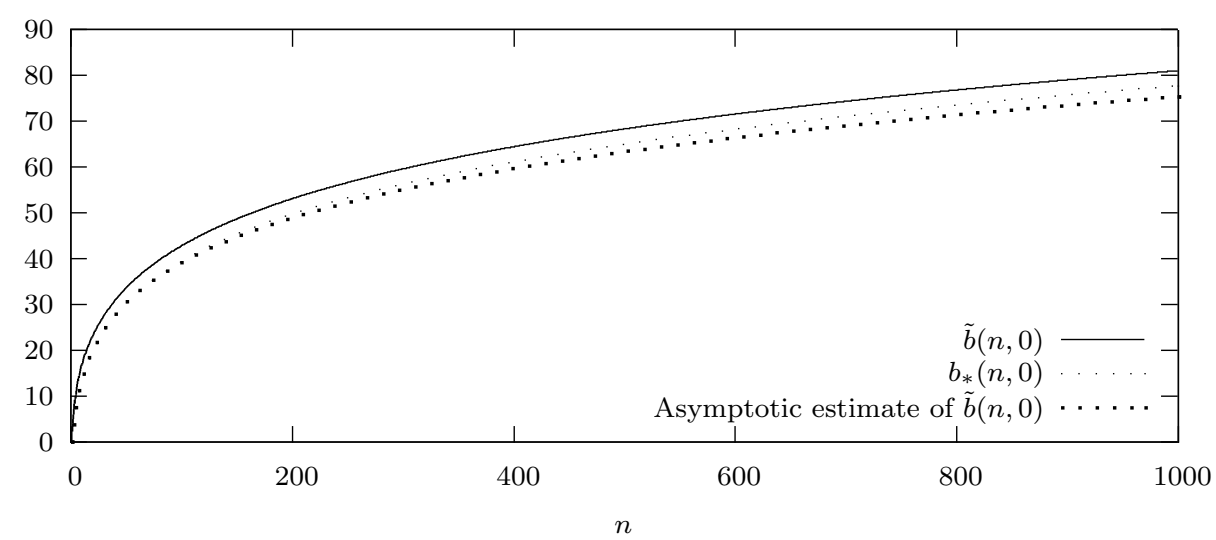

Figure 2: Numerical values of $\tilde{b}(n, 0), b_{*}(n, 0)$, and asymptotic estimate of $\tilde{b}(n, 0)$ with $p=0.5$.

where

$$
A_{*}(-1)=\sum_{\ell=2}^{\infty} \frac{\lceil\log (\ell+1)\rceil}{\ell(\ell-1)}
$$

if $\log p / \log q$ is irrational. If $\log p / \log q=r / s$ is rational, the constant $A_{*}(-1)$ in $(21)$ must be replaced by the oscillatory function

$$
A_{*}(-1)+\sum_{k=-\infty, k \neq 0}^{\infty} A_{*}\left(-1+\frac{2 k \pi i r}{\log p}\right) e^{2 k \pi i r \log _{p} n}
$$

where

$$
A_{*}(s)=\sum_{n \geqslant 2} \frac{\lceil\log (n+1)\rceil}{n !} \Gamma(n+s) .
$$

By analyzing (19) and (20) for $n \rightarrow \infty$ we can show that the difference $a(n, \infty)-a(n, d)$ is $O(\log n)$, and more precisely

$$
\tilde{a}(n, 0)=a(n, \infty)-a(n, 0) \sim-\frac{A_{*}(-1)}{h \log p} \log n .
$$

Again if $\log p / \log q$ is rational we must replace $A_{*}(-1)$ by the Fourier series in $(22)$.

\subsection{Numerical Data}

To confirm our results, we numerically computed some of the quantities discussed above. In Figure 2, we plot the values of $\tilde{b}(n, 0)$ (defined in $(13)), b_{*}(n, 0)$ (defined by $(16)$ and (17)), and our asymptotic estimate of $\tilde{b}(n, 0)$ shown in Theorem 1 , with $p=1 / 2$. We computed this asymptotic estimate up to the $\log n$ term without the periodic function $\Psi(\cdot)$, that is,

$$
\frac{1}{2 h \log (1 / p)} \log ^{2} n-\frac{d}{h} \log n+\left[\frac{1}{2 h}-\frac{1}{h \log p}\left(\gamma+1+\frac{h_{2}}{2 h}\right)\right] \log n .
$$


Table 1: Numerical values of $\tilde{b}(n, 0)$ and $b_{*}(n, 0)$

\begin{tabular}{|c||c|c|c||c|c|c|}
\hline \multicolumn{1}{|c||}{} & \multicolumn{4}{c||}{$p=1 / 2$} & \multicolumn{3}{c|}{$p=1 / 3$} \\
\hline$n$ & $\tilde{b}(n, 0)$ & $b_{*}(n, 0)$ & $\tilde{b}(n, 0)-b_{*}(n, 0)$ & $\tilde{b}(n, 0)$ & $b_{*}(n, 0)$ & $\tilde{b}(n, 0)-b_{*}(n, 0)$ \\
\hline 1 & 0 & 0 & 0 & 0 & 0 & 0 \\
2 & 4 & 4 & 0 & 4.5 & 4.5 & 0 \\
3 & 6 & 5 & 1 & 7 & 5 & 2 \\
4 & 8.321 & 6.696 & 1.625 & 9.507 & 6.248 & 3.259 \\
5 & 10.305 & 8.121 & 2.184 & 11.652 & 7.369 & 4.283 \\
10 & 16.472 & 13.267 & 3.205 & 17.659 & 11.466 & 6.193 \\
20 & 23.034 & 19.752 & 3.282 & 22.521 & 16.260 & 6.261 \\
30 & 27.406 & 24.124 & 3.282 & 25.802 & 19.494 & 6.308 \\
50 & 33.489 & 30.207 & 3.282 & 30.150 & 23.891 & 6.259 \\
100 & 42.724 & 39.442 & 3.282 & 36.849 & 30.550 & 6.298 \\
\hline
\end{tabular}

We also present the numerical values of $\tilde{b}(n, 0), b_{*}(n, 0)$, and their difference for $p=1 / 2$ and $p=1 / 3($ then $\log (p) / \log (q)$ is irrational) in Table 1 , which confirms that $\tilde{b}(n, 0) \sim$ $b_{*}(n, 0)$, and suggests that the difference is $O(1)$ for $n \rightarrow \infty$ (which we shall establish analytically).

\section{Analysis}

We first discuss some exact solutions of recurrence (4) for small values of $n$ and arbitrary $d$, then we prove our Theorem 1 , and finally provide solutions of (4) for other ranges of $(n, d)$, where $d \rightarrow \infty$.

\subsection{Some Exact Solutions}

We first consider (4) for small values of $n$ and arbitrary $d$. Using (5) we rewrite (4) as

$$
b(n, d)=n+\sum_{k=2}^{n}\left(\begin{array}{l}
n \\
k
\end{array}\right) p^{k} q^{n-k} b(k, d-1)+\sum_{k=0}^{n-2}\left(\begin{array}{l}
n \\
k
\end{array}\right) p^{k} q^{n-k} b(n-k, k+d-1) .
$$

When $n=2$, (23) yields $b(2, d)=2+\left(p^{2}+q^{2}\right) b(2, d-1)$ and since $b(2,0)=0$ we have

$$
b(2, d)=\frac{1}{p q}+\left(2-\frac{1}{p q}\right)\left(p^{2}+q^{2}\right)^{d-1} \text { for } d \geqslant 1 .
$$

Note that $b(2, \infty)=(p q)^{-1}$ by (8). Setting $n=3$ in (23) and solving the resulting equation leads to, after some calculation,

$$
b(3, d)=\frac{2}{p q}+\frac{3}{p q}\left(2 p q^{2}-1\right)\left(p^{2}+q^{2}\right)^{d}+\left(2+\frac{1}{p q}-6 q\right)\left(p^{3}+q^{3}\right)^{d} \text { for } d \geqslant 0 .
$$


We can then continue solving $b(n, d)$ for increasing $n$, and it is clear that $b(n, d)$ will have the form

$$
b(n, d)=b(n, \infty)-\sum_{J=2}^{n}\left(p^{J}+q^{J}\right)^{d} B(n, J),
$$

where $b(n, \infty)$ is the trie path length in (8) and (9). It follows that $b(n, d)-b(n, \infty)=$ $O\left[\left(p^{2}+q^{2}\right)^{d}\right]$ for $n$ fixed and $d \rightarrow \infty$. We can characterize the double sequence $B(n, J)$ by using (24) in (23) and equating coefficients of $\left(p^{J}+q^{J}\right)^{d}$. For $J \geqslant 2$ this leads to

$$
B(n, J)=\frac{1}{p^{J}+q^{J}} \sum_{k=J}^{n}\left(\begin{array}{l}
n \\
k
\end{array}\right)\left(p^{k} q^{n-k}+q^{k}\left[p\left(p^{J}+q^{J}\right)\right]^{n-k}\right) B(k, J) .
$$

From (6) and (24) we find that

$$
b(n, \infty)+\sum_{J=2}^{n+1} B(n+1, J)=b(n+1, \infty)+\sum_{J=2}^{n} B(n, J)\left(p^{J}+q^{J}\right) .
$$

We can transform (25) into another equation by introducing the generating function

$$
\mathcal{F}_{J}(z)=\sum_{n=0}^{\infty} B(n, J) \frac{z^{n}}{n !}=\sum_{n=J}^{\infty} B(n, J) \frac{z^{n}}{n !} .
$$

Using (27) in (25) leads to the functional equation

$$
\mathcal{F}_{J}(z)=\frac{1}{p^{J}+q^{J}}\left(\mathcal{F}_{J}(p z) e^{q z}+\mathcal{F}_{J}(q z) e^{p\left(p^{J}+q^{J}\right) z}\right) \text { for } J \geqslant 2 .
$$

Then if $\mathcal{F}_{J}(z)=e^{z} \mathcal{G}_{J}(z)$ we obtain

$$
\left(p^{J}+q^{J}\right) \mathcal{G}_{J}(z)=\mathcal{G}_{J}(p z)+\mathcal{G}_{J}(q z) e^{p\left(p^{J}+q^{J}-1\right) z} .
$$

Again this appears difficult to solve explicitly (however, see [10]).

We can take the analysis somewhat further in the symmetric case where $p=q=1 / 2$, as then (28) simplifies to

$$
\mathcal{G}_{J}(z) 2^{1-J}=\mathcal{G}_{J}\left(\frac{z}{2}\right)\left(1+\exp \left[\left(2^{1-J}-1\right) \frac{z}{2}\right]\right) .
$$

Solving (28) and inverting the transform over $z$ leads ultimately to

$$
B(n, J)=\frac{B(J, J)}{J !} \frac{n !}{2 \pi i} \oint \frac{e^{z}}{z^{n+1-J}} \prod_{L=0}^{\infty}\left(\frac{1+e^{\left(2^{1-J}-1\right) 2^{-L-1} z}}{2}\right) d z .
$$

Thus the double sequence $B(n, J)$ is known up to the single sequence $B(J, J)$. To determine $B(J, J)$ we must still use (26). Thus putting (30) in (26) will lead to a single variable recurrence for $B(J, J)$. 
Next we return to general $p, q$ and estimate $B(n, 2)$ in $(25)$ for $n \rightarrow \infty$. Let us set $C(n)=B(n, 2)$ and we recall that, by $(24)$,

$$
\tilde{b}(n, d) \sim C(n)\left(p^{2}+q^{2}\right)^{d} ; \quad d \rightarrow \infty, \quad n=O(1) .
$$

While we mainly want to estimate $\tilde{b}(n, d)$ for $n \rightarrow \infty$ and $d=O(1)$, it is useful to try to understand the full asymptotic structure of $\tilde{b}(n, d)$, for $n$ and/or $d$ large.

We thus examine how (31) behaves when $n$ also becomes large. Setting $J=2$ in (25) leads to

$$
\left(p^{2}+q^{2}\right) C(n)=\sum_{k=2}^{n}\left(\begin{array}{l}
n \\
k
\end{array}\right) p^{k} q^{n-k} C(k)+\sum_{k=0}^{n-2}\left(\begin{array}{l}
n \\
k
\end{array}\right) p^{k} q^{n-k}\left(p^{2}+q^{2}\right)^{k} C(n-k)
$$

for $n \geqslant 3$ with $C(2)=\left(p^{-1} q^{-1}-2\right) /\left(p^{2}+q^{2}\right)=(p q)^{-1}$.

We argue intuitively that $C(n)$ will behave algebraically for $n \rightarrow \infty$ (we shall prove this fact shortly). Then we use the fact that the "kernel" in (32) behaves

$$
\left(\begin{array}{l}
n \\
k
\end{array}\right) p^{k} q^{n-k} \rightarrow \delta(k-n p), \quad n \rightarrow \infty
$$

where $\delta(\cdot)$ is the delta function. Then for algebraically or logarithmically varying smooth $f(k)$ (for $k \rightarrow \infty$ ) we have (see [6,9] for rigorous proofs)

$$
\sum_{k=0}^{n}\left(\begin{array}{l}
n \\
k
\end{array}\right) p^{k} q^{n-k} f(k)=f(n p)+O\left(n f^{\prime \prime}(n p)\right), n \rightarrow \infty .
$$

Then the term involving $\left(p^{2}+q^{2}\right)^{k} C(n-k)$ will lead to terms that are exponentially smaller than those arising from $C(k)$, and (32) may be replaced by the asymptotic relation

$$
C(n)\left(p^{2}+q^{2}\right) \sim C(n p), n \rightarrow \infty .
$$

A general solution to (33) has the form

$$
C(n)=n^{\nu} \bar{C}(n)
$$

where $\bar{C}(n p)=\bar{C}(n)$ and $p^{\nu}=p^{2}+q^{2}$ so that

$$
\nu=\frac{\log \left(p^{2}+q^{2}\right)}{\log p}>0 .
$$

Thus $\bar{C}(\cdot)$ is a periodic function of $\log _{p} n$ of period 1 , which we can write as the Fourier series

$$
\bar{C}(n)=c^{(0)}(p)+\sum_{\ell=-\infty, \ell \neq 0}^{\infty} c^{(\ell)}(p) e^{2 \pi i \ell \log _{p} n} .
$$


It again appears difficult to identify explicitly the Fourier coefficients $c^{(\ell)}(p)$, but we can do this in the symmetric case $p=q=1 / 2$. Then we set $\sum_{n=0}^{\infty} C(n) z^{n} / n !=\mathcal{F}_{2}(z)$ as in (27) and from (30) obtain

$$
C(n)=\frac{2 n !}{2 \pi i} \oint \frac{e^{z}}{z^{n-1}} \prod_{\ell=1}^{\infty}\left(\frac{1+e^{-z 2^{-\ell-1}}}{2}\right) d z .
$$

To obtain the large $n$ behavior of the integral in (37) we first expand the integral for $z \rightarrow \infty$ and apply a depoissonization argument. Setting $\ell=\log _{2} z+J$ we have $2^{\ell}=2^{J} z$ and

$$
\begin{aligned}
& \prod_{\ell=1}^{\infty}\left(\frac{1+e^{-z 2^{-\ell-1}}}{2}\right) \\
= & \exp \left[\sum_{\ell=1}^{\infty} \log \left(\frac{1+e^{-z 2^{-\ell-1}}}{2}\right)\right] \\
= & \exp \left[\sum_{J=1-\log _{2} z}^{\infty} \log \left(\frac{1+e^{-2^{-J-1}}}{2}\right)\right] \\
= & \exp \left[\sum_{J=0}^{\infty} \log \left(\frac{1+e^{-2^{-J-1}}}{2}\right)+\sum_{J=1}^{\log _{2} z-1} \log \left(\frac{1+e^{-2^{J-1}}}{2}\right)\right] \\
\sim & \exp \left[\log \left(\frac{1}{2}\right)\left(\log _{2} z-1\right)+\sum_{J=0}^{\infty} \log \left(\frac{1+e^{-2^{-J-1}}}{2}\right)+\sum_{J=1}^{\infty}\left(1+e^{-2^{J-1}}\right)\right] \\
= & \frac{2}{z} K_{*}
\end{aligned}
$$

where

$$
K_{*}=\prod_{J=0}^{\infty}\left(\frac{1+e^{-2^{-J-1}}}{2}\right) \prod_{J=1}^{\infty} \exp \left(1+e^{-2^{J-1}}\right)=1 .
$$

Thus $C(n) \sim 4 n ! /(n-1) !=4 n$ as $n \rightarrow \infty$. This shows that $c^{(0)}(1 / 2)=4$ and a more careful calculation can be used to identify the other Fourier coefficients $c^{(\ell)}(1 / 2)$ in $(36)$ (then we would set $\ell=\left\lfloor\log _{2} z\right\rfloor+J=\log _{2} z+J-\left\{\log _{2} z\right\}$ so that $2^{\ell}=2^{J} z 2^{-\left\{\log _{2} z\right\}}$. We omit the details.

In Table 2 we consider various values of $p$ and estimate $\bar{C}(n) \approx c^{(0)}(p)$ numerically, by computing $C(n) n^{-\nu}$ from (32), for large $n$. This shows that as a function of $p,\left|c^{(0)}(p)\right|$ is minimal when $p$ is between 0.6 and 0.7 , and becomes large as either $p \rightarrow 0$ or $p \rightarrow 1$. For $p \rightarrow 0$ the oscillatory terms in (36) become more numerically significant. Table 2 indicates this when $p=0.1$, by giving a range of values of $C(n) n^{-\nu}$.

To justify the approximation in (33) we first inductively show that for all $n$

$$
C(n) \leqslant A n^{\nu+\epsilon}
$$


Table 2: Values of the zeroth Fourier coefficient.

\begin{tabular}{|c|c|}
\hline$p$ & $\left.C(n) n^{-\nu}\right|_{n \rightarrow \infty} \approx c^{(0)}(p)$ \\
\hline 0.5 & 4 \\
0.4 & 5.664 \\
0.3 & 9.728 \\
0.25 & 14.03 \\
0.2 & 22.5 \\
0.1 & 98 to 105 \\
& \\
0.6 & 3.331 \\
0.7 & 3.276 \\
0.75 & 3.479 \\
0.8 & 3.903 \\
0.9 & 6.423 \\
\hline
\end{tabular}

for all $\epsilon>0$ and $A>0$. By isolating the terms in the sums in (32) with $k=n$ and $k=0$ we obtain, for $n>2$,

$$
C(n)=\frac{1}{p^{2}+q^{2}-p^{n}-q^{n}}\left[\sum_{k=2}^{n-1}\left(\begin{array}{l}
n \\
k
\end{array}\right) p^{k} q^{n-k} C(k)+\sum_{k=1}^{n-2}\left(\begin{array}{l}
n \\
k
\end{array}\right) p^{k} q^{n-k}\left(p^{2}+q^{2}\right)^{k} C(n-k)\right] .
$$

Assuming inductively that (38) holds for $C(k)$ for $k=1,2, \cdots, n-1$ we then have

$$
\begin{aligned}
\sum_{k=2}^{n-1}\left(\begin{array}{l}
n \\
k
\end{array}\right) p^{k} q^{n-k} C(k) & \leqslant \sum_{k=2}^{n-1}\left(\begin{array}{l}
n \\
k
\end{array}\right) p^{k} q^{n-k} A k^{\nu+\epsilon} \\
& \leqslant A(n p)^{\nu+\epsilon} .
\end{aligned}
$$

Using a similar estimate for the second sum in (39) we are led to

$$
\begin{aligned}
C(n) & \leqslant \frac{A}{p^{2}+q^{2}-p^{n}-q^{n}}\left[(n p)^{\nu+\epsilon}+n^{\nu+\epsilon}\left(p\left(p^{2}+q^{2}\right)+q\right)^{n}\right] \\
& =A n^{\nu+\epsilon}\left[\frac{p^{2}+q^{2}}{p^{2}+q^{2}-p^{n}-q^{n}} p^{\epsilon}+\frac{\left(p\left(p^{2}+q^{2}\right)+q\right)^{n}}{p^{2}+q^{2}-p^{n}-q^{n}}\right],
\end{aligned}
$$

as $C(n-k) \leqslant A(n-k)^{\nu+\epsilon} \leqslant A n^{\nu+\epsilon}$ and $p^{\nu}=p^{2}+q^{2}$. Since $p\left(p^{2}+q^{2}\right)+q<p+q=1$, the second term in (40) is asymptotically negligible for $n$ large and (38) follows by induction.

We have thus obtained some exact expressions for $b(n, d)$ for small values of $n$, a general asymptotic result for $d \rightarrow \infty$ with $n=O(1)$, and then examined how this result behaves when $n$ also becomes large. However, this cannot be used to infer the behavior of $b(n, d)$ for $n \rightarrow \infty$ with $d=O(1)$, which we examine next. 


\subsection{Main Asymptotic Result for $b(n, d)$}

We first give an intuitive derivation of the asymptotics of $b(n, d)$ for fixed $d \geqslant 0$ and $n \rightarrow \infty$, and in particular of $b(n, 0)$. Starting from (14) we again argue that the second sum is negligible for $n \rightarrow \infty$ and that the first is asymptotic to $\tilde{b}(n p, d-1)$ so that (14) becomes

$$
\tilde{b}(n, d) \sim \tilde{b}(n p, d-1), n \rightarrow \infty
$$

and, in particular,

$$
\tilde{b}(n, 1) \sim \tilde{b}(n p, 0), n \rightarrow \infty
$$

which when added to (15) leads to

$$
\tilde{b}(n+1,0)-\tilde{b}(n p, 0) \sim b(n+1, \infty)-b(n, \infty) .
$$

The right side of (43) may be estimated from (10) or by (9). Using (9) we can show that term by term differentiating of the asymptotic series in (10) is permissible, and thus (43) becomes, for $n \rightarrow \infty$,

$$
\tilde{b}(n+1,0)-\tilde{b}(n p, 0)=\frac{1}{h} \log n+\frac{1}{h}\left(\gamma+1+\frac{h_{2}}{2 h}\right)+\frac{1}{h} \psi\left(\log _{p} n\right)+o(1),
$$

where $\psi(\cdot)$ is the periodic function

$$
\psi(x)=\sum_{k=-\infty, k \neq 0}^{\infty}\left[1+\frac{2 k \pi i r}{\log p}\right] \Gamma\left(-\frac{2 k \pi i r}{\log p}\right) e^{2 k \pi i r x},
$$

where we note that $\psi$ and $\Phi$ are related by $\psi(x)=\Phi(x)+(\log p)^{-1} \Phi^{\prime}(x)$.

Now (44) suggests that $\tilde{b}(n, 0)$ admits an asymptotic expansion of the form

$$
\tilde{b}(n, 0)=A \log ^{2} n+B \log n+C+o(1), n \rightarrow \infty
$$

and then

$$
\tilde{b}(n+1,0)-\tilde{b}(n p, 0)=-2 A(\log p) \log n-A \log ^{2} p-B \log p+o(1) .
$$

Comparing (44) to (47) we conclude that $A=-(2 h \log p)^{-1}$ and then

$$
B=\frac{1}{2 h}-\frac{1}{h \log p}\left[\gamma+1+\frac{h_{2}}{2 h}+\psi\left(\log _{p} n\right)\right] .
$$

We have thus formally derived the result in Theorem 1 for $\tilde{b}(n, 0)$. For any fixed $d>0$ we can extend this argument by asymptotically solving (41) by an expansion of the form

$$
\tilde{b}(n, d)=A(d) \log ^{2} n+B(d) \log n+C(d)+o(1)
$$

to find from (41) that $A(d)=A(d-1)$ and $B(d)=B(d-1)+2 \log p A(d-1)$. Then using (49) in (43) or (44) we find that $A(d)=A(0)=-(2 h \log p)^{-1}$ and $B(d)-B(d-1)=$ $2 \log p A(d-1)=-h^{-1}$ so that $B(d)=B(0)-h^{-1} d$, where $B(0)=B$ is as in (48). 
We proceed to provide a rigorous derivation of the theorem. Using arguments completely analogous to (38)-(40), we can inductively establish the bound

$$
\tilde{b}(n, d) \leqslant A_{0} n^{\nu+\epsilon}\left(p^{2}+q^{2}\right)^{d} ; n \geqslant 2, d \geqslant 0
$$

where again $\nu$ is given by (35). When $n=2$ we have (exactly)

$$
\tilde{b}(2, d)=\left(\frac{1}{p q}-2\right)\left(p^{2}+q^{2}\right)^{d-1},
$$

so (50) clearly holds. Assuming that (50) holds for all $(N, D)$ with $N+D<n+d$ we can estimate the first sum in the right side of (13) by

$$
\begin{aligned}
\sum_{k=0}^{n-2}\left(\begin{array}{l}
n \\
k
\end{array}\right) p^{k} q^{n-k} \tilde{b}(k, d-1) & \leqslant A_{0} \sum_{k=0}^{n} k^{\nu+\epsilon}\left(p^{2}+q^{2}\right)^{d-1} p^{k} q^{n-k}\left(\begin{array}{l}
n \\
k
\end{array}\right) \\
& \leqslant A_{0}(n p)^{\nu+\epsilon}\left(p^{2}+q^{2}\right)^{d-1} \\
& =A_{0} n^{\nu+\epsilon}\left(p^{2}+q^{2}\right)^{d}
\end{aligned}
$$

and the second sum (14) by

$$
\begin{aligned}
\sum_{k=0}^{n-2}\left(\begin{array}{l}
n \\
k
\end{array}\right) p^{k} q^{n-k} \tilde{b}(n-k, k+d-1) & \leqslant A_{0} \sum_{k=0}^{n}(n-k)^{\nu+\epsilon}\left(p^{2}+q^{2}\right)^{k+d-1} p^{k} q^{n-k}\left(\begin{array}{l}
n \\
k
\end{array}\right) \\
& \leqslant A_{0} n^{\nu+\epsilon}\left(p^{2}+q^{2}\right)^{d-1} \sum_{k=0}^{n}\left(\begin{array}{l}
n \\
k
\end{array}\right)\left[p\left(p^{2}+q^{2}\right)\right]^{k} q^{n-k} \\
& =A_{0} n^{\nu+\epsilon}\left(p^{2}+q^{2}\right)^{d-1}\left[q+p\left(p^{2}+q^{2}\right)\right]^{n}
\end{aligned}
$$

which is $o(\tilde{b}(n, d))$ (by an exponentially small factor).

Now let $b_{\text {diff }}(n, d)=\tilde{b}(n, d)-b_{*}(n, d)$. Then from (14)-(17) we see that

$$
b_{\text {diff }}(n, d)=\sum_{k=0}^{n}\left(\begin{array}{l}
n \\
k
\end{array}\right) p^{k} q^{n-k} b_{\text {diff }}(k, d-1)+\sum_{k=0}^{n}\left(\begin{array}{l}
n \\
k
\end{array}\right) p^{k} q^{n-k} \tilde{b}(n-k, k+d-1)
$$

with $b_{\text {diff }}(n+1,0)=b_{\text {diff }}(n, 1)$. Using an inductive argument analogous to that used to obtain (50) we can show that $b_{\text {diff }}(n, d)$ is $O(1)$, since the second sum in (51) may be estimated to be exponentially small for $n \rightarrow \infty$. We shall show below that $b_{*}(n, d)=$ $O\left(\log ^{2} n\right)$ for a fixed $d$ and $n \rightarrow \infty$, and hence $\tilde{b}(n, d) \sim b_{*}(n, d)$.

We proceed to analyze (16), with (17), and thus re-establish Theorem 1. Introducing the exponential generating function

$$
B_{d}^{*}(z)=\sum_{n=2}^{\infty} b_{*}(n, d) \frac{z^{n}}{n !}=e^{z} A_{d}(z)
$$

where $b_{*}(n, d)$ is defined from (16), we find that

$$
B_{d}^{*}(z)=B_{d-1}^{*}(p z) e^{q z}
$$


or, since $A_{d}(z)=B_{d}^{*}(z) e^{-z}$,

$$
A_{d}(z)=A_{d-1}(p z)
$$

This can be solved by iteration to yield

$$
A_{d}(z)=A_{0}\left(p^{d} z\right)
$$

Then setting

$$
\mathcal{G}_{*}(z)=\sum_{n=2}^{\infty} b(n, \infty) \frac{z^{n}}{n !}
$$

and noting that

$$
\sum_{n=1}^{\infty} b_{*}(n+1,0) \frac{z^{n}}{n !}=\frac{d}{d z} B_{0}^{*}(z)
$$

(17) leads to

$$
\frac{d}{d z} B_{0}^{*}(z)-B_{1}^{*}(z)=-\mathcal{G}_{*}(z)+\mathcal{G}_{*}^{\prime}(z)
$$

If $\mathcal{G}_{*}(z)=e^{z} \tilde{\mathcal{G}}(z)$, from the integral representation in (9) we conclude that the Mellin transform of $\tilde{\mathcal{G}}(z)$ is

$$
\int_{0}^{\infty} \tilde{\mathcal{G}}(z) z^{s-1} d z=\frac{\Gamma(s+1)}{1-p^{-s}-q^{-s}},
$$

Using the definitions of $A_{d}(\cdot)$ and $\tilde{\mathcal{G}}(\cdot),(54)$ becomes

$$
A_{0}^{\prime}(z)+A_{0}(z)-A_{0}(p z)=\tilde{\mathcal{G}}^{\prime}(z) .
$$

We introduce the negative Mellin transform of $A_{0}(z)$

$$
\mathcal{M}(s)=-\int_{0}^{\infty} A_{0}(z) z^{s-1} d z
$$

and use (57) in (56) to obtain the functional equation

$$
-(s-1) \mathcal{M}(s-1)+\left(1-p^{-s}\right) \mathcal{M}(s)=\frac{(s-1) \Gamma(s)}{1-p^{1-s}-q^{1-s}} .
$$

Next we set

$$
\mathcal{M}(s)=\Gamma(s) \mathcal{N}(s)
$$

with which (58) becomes

$$
-\mathcal{N}(s-1)+\left(1-p^{-s}\right) \mathcal{N}(s)=\frac{s-1}{1-p^{1-s}-q^{1-s}} .
$$

To solve (60) we let

$$
\mathcal{N}(s)=\prod_{k=0}^{\infty}\left[\frac{1-p^{k+2}}{1-p^{k-s}}\right] \mathcal{N}_{1}(s)
$$


and then (60) becomes

$$
\mathcal{N}_{1}(s)-\mathcal{N}_{1}(s-1)=\prod_{k=1}^{\infty}\left[\frac{1-p^{k-s}}{1-p^{k+1}}\right] \frac{s-1}{1-p^{1-s}-q^{1-s}} .
$$

Now, for $s \rightarrow-\infty$ the right side of $(62)$ behaves as $(s-1) \prod_{k=1}^{\infty}\left(1-p^{k+1}\right)^{-1}$, with an exponentially small error. Letting

$$
\mathcal{N}_{1}(s)=\frac{s(s-1)}{2} \prod_{k=1}^{\infty}\left(\frac{1}{1-p^{k+1}}\right)+\mathcal{N}_{2}(s)
$$

the equation for $\mathcal{N}_{2}(\cdot)$ becomes

$$
\mathcal{N}_{2}(s)-\mathcal{N}_{2}(s-1)=\frac{s-1}{\prod_{k=1}^{\infty}\left(1-p^{k+1}\right)}\left[\frac{1}{1-p^{1-s}-q^{1-s}} \prod_{k=1}^{\infty}\left(1-p^{k-s}\right)-1\right]
$$

whose right hand side is, unlike that of (62), exponentially small for $s \rightarrow-\infty$. The solution to $(64)$ is

$$
\mathcal{N}_{2}(s)=\mathcal{N}_{2}(-\infty)+\sum_{i=0}^{\infty}\left[\frac{\prod_{k=1}^{\infty}\left(1-p^{k-s+i}\right)}{1-p^{1+i-s}-q^{1+i-s}}-1\right] \frac{s-1-i}{\prod_{k=1}^{\infty}\left(1-p^{k+1}\right)}
$$

From (52) we see that $A_{d}(z)=O\left(z^{2}\right)$ as $z \rightarrow 0$ so that $\mathcal{M}(s)$ in (57) must be analytic at $s=-1$. From (59) we then conclude that $\mathcal{N}(-1)=0$. From $(61)$ we have $\mathcal{N}_{1}(-1)=0$ and from (63) and (65) we thus obtain an expression for $\mathcal{N}_{2}(-\infty)$ :

$$
\mathcal{N}_{2}(-\infty) \prod_{k=1}^{\infty}\left(1-p^{k+1}\right)+1-\sum_{i=0}^{\infty}(i+2)\left[\frac{\prod_{k=1}^{\infty}\left(1-p^{k+i+1}\right)}{1-p^{2+i}-q^{2+i}}-1\right]=0 .
$$

We have thus obtained the final expression for $\mathcal{M}(s)$ in (59) as

$$
\mathcal{M}(s)=\frac{\Gamma(s)}{\prod_{L=0}^{\infty}\left(1-p^{L-s}\right)}\left(\frac{s(s-1)}{2}+\beta+\sum_{i=0}^{\infty}(s-i-1)\left[\frac{\prod_{k=1}^{\infty}\left(1-p^{k-s+i}\right)}{1-p^{1+i-s}-q^{1+i-s}}-1\right]\right),
$$

where

$$
\beta=\mathcal{N}_{2}(-\infty) \prod_{k=1}^{\infty}\left(1-p^{k+1}\right)
$$

can be computed from (66). Inverting the transform in (57) we obtain the generating function of $b_{*}$ as

$$
\sum_{n=0}^{\infty} \frac{z^{n}}{n !} b_{*}(n, d)=\frac{-1}{2 \pi i} \int_{B r}\left(p^{d} z\right)^{-s} \mathcal{M}(s) d s .
$$

The final step is to expand $b_{*}(n, d)(\sim \tilde{b}(n, d))$ for $n \rightarrow \infty$ with $d$ fixed. Inverting the generating function over $z$ can be done asymptotically by a standard depoissonization 
argument, which amounts to simply replacing $z$ by $n$ for large $n$. Thus we need only evaluate the integral in (68) for $z$ large and positive. The function $\mathcal{M}(s)$ in (67) has a triple pole at $s=0$, and there are other double poles on the imaginary $s$-axis if $1-p^{1-s}-q^{1-s}$ has zeros there, which occurs only if $\log p / \log q$ is rational, say $r / t$ where $r$ and $t$ are integers. First we compute the contribution from $s=0$. Using the expansion $\Gamma(s)=$ $\left[1-\gamma s+O\left(s^{2}\right)\right] / s$ as $s \rightarrow 0$, with $\gamma$ being the Euler constant, (67) becomes

$$
\begin{aligned}
\mathcal{M}(s)= & \frac{1}{s}\left[1-\gamma s+O\left(s^{2}\right)\right]\left(1-p^{-s}\right)^{-1} \prod_{L=1}^{\infty}\left(1-p^{L-s}\right)^{-1} \\
& \times\left(\frac{s-1}{1-p^{1-s}-q^{1-s}} \prod_{k=1}^{\infty}\left(1-p^{k-s}\right)-(s-1)+\frac{s(s-1)}{2}+\beta\right. \\
& \left.+\sum_{i=1}^{\infty}(s-i-1)\left[\frac{\prod_{k=1}^{\infty}\left(1-p^{k-s+i}\right)}{1-p^{1+i-s}-q^{1+i-s}}-1\right]\right) .
\end{aligned}
$$

Now

$$
1-p^{-s}=s \log p-\frac{1}{2} s^{2}(\log p)^{2}+O\left(s^{3}\right)
$$

and

$$
1-p^{1-s}-q^{1-s}=-h s-\frac{h_{2}}{2} s^{2}+O\left(s^{3}\right) .
$$

Also, using the expression in (66) to compute $\beta+1$ the expansion of (69) for $s \rightarrow 0$ becomes

$$
\begin{aligned}
\mathcal{M}(s) & =\frac{1}{s^{3}} \frac{1-\gamma s}{\log p}\left[1+\frac{s}{2} \log p+O\left(s^{2}\right)\right]\left\{\frac{1-s}{h}\left[1-\frac{h_{2}}{2 h} s+O\left(s^{2}\right)\right]+O\left(s^{2}\right)\right\} \\
& =\frac{1}{s^{3}} \frac{1}{h \log p}+\frac{1}{s^{2}}\left[-\frac{\gamma}{h \log p}-\frac{1}{h \log p}\left(1+\frac{h_{2}}{2 h}\right)+\frac{1}{2 h}\right]+O\left(\frac{1}{s}\right) .
\end{aligned}
$$

It follows that the integrand $p^{-d s} z^{-s} \mathcal{M}(s)$ in (68) has the residue

$$
\operatorname{Res}_{s=0}\left\{p^{-d s} z^{-s} \mathcal{M}(s)\right\}=\frac{1}{2} \frac{\log ^{2} z}{h \log p}+\frac{d}{h} \log z+\log z\left[\frac{1}{\log p}\left(\frac{\gamma+1}{h}+\frac{h_{2}}{2 h^{2}}\right)-\frac{1}{2 h}\right]+O(1)
$$

where the $O(1)$ refers to terms that are $O(1)$ for $z \rightarrow \infty$, and these can be evaluated by explicitly computing the $O\left(s^{-1}\right)$ term(s) in (70). Then the expansion of $\tilde{b}(n, d) \sim b_{*}(n, d)$ follows by setting $z=n$ in (71), and we have thus regained the formula in Theorem 1 . If $\log p / \log q$ is rational we must also compute the contribution from the double poles along the imaginary axis at such points $p^{-s}=q^{-s}=1$ and $p^{1-s}+q^{1-s}=1$. These poles lead to the oscillatory terms in Theorem 1 , as can be seen by computing their residues from (67).

We have thus established Theorem 1 rigorously, though the intuitive derivation in (41)-(49) is much simpler, and more revealing of the basic asymptotic structure of the equations (14) and (15). 


\subsection{Other Asymptotic Ranges}

Here we briefly discuss $\tilde{b}(n, d)$ when $n$ and $d$ are simultaneously large, and try to identify what ranges of $n$ and $d$ lead to different asymptotic expansions. We recall that (31) applies for $n$ fixed and $d \rightarrow \infty$, while Theorem 1 applies for $d$ fixed and $n \rightarrow \infty$. We confine ourselves here to an intuitive discussion.

The form of the expansion in (31) (with $C(n)$ given by (34) and (36)) suggests that an important scale is $n, d \rightarrow \infty$ with $d-\log _{1 / p}(n)=O(1)$. Note that then the algebraic growth of $n^{\nu}$ as $n \rightarrow \infty$ is balanced by the geometric decay of $\left(p^{2}+q^{2}\right)^{d}$ in (31). We introduce the new variable $\xi$ with

$$
d=\frac{\log n}{\log (1 / p)}+\xi, \quad \xi=O(1)
$$

with

$$
\tilde{b}(n, d)=\mathcal{B}(n, \xi)=\mathcal{B}\left(n, d-\log _{1 / p}(n)\right)
$$

and we note that

$$
\tilde{b}(n p, d-1)=\mathcal{B}(n p, \xi) .
$$

We again argue that for $n \rightarrow \infty$ the second sum in (14) is negligible and approximate (14) by

$$
\tilde{b}(n, d)=\tilde{b}(n p, d-1)+O\left[n \tilde{b}^{\prime \prime}(n p, d-1)\right] .
$$

In view of (73) and (74) a general asymptotic solution of (75) is any function that satisfies $\mathcal{B}(n, \xi)=\mathcal{B}(n p, \xi)$ which we can write as a Fourier series, with

$$
\mathcal{B}(n, \xi)=\mathcal{B}_{0}(\xi)+\sum_{\ell=-\infty, \ell \neq 0}^{\infty} e^{2 \pi i \ell \log _{p}(n)} \mathcal{B}_{\ell}(\xi)
$$

Thus (76) gives an approximation to $\tilde{b}(n, d)$ for $n, d \rightarrow \infty$ with $\xi=O(1)$, but we cannot explicitly determine the Fourier coefficients $\mathcal{B}_{\ell}(\xi)$, which are now functions of $\xi$. If we require $\mathcal{B}(n, \xi)$ to asymptotically match to $(31)$, we would equate the large $n$ behavior (31) to the expansion of $\mathcal{B}(n, \xi)$ for $\xi \rightarrow+\infty$, and this yields

$$
\mathcal{B}_{0}(\xi) \sim c^{(0)} e^{\xi \log \left(p^{2}+q^{2}\right)}, \quad \xi \rightarrow+\infty
$$

and a similar matching condition can be obtained for $\mathcal{B}_{\ell}(\xi)$ for $\ell \neq 0$, by comparing (76) and (34) with (36). Thus (77) shows that $\mathcal{B}_{0}(\xi)$ will decay exponentially for $\xi \rightarrow+\infty$.

Next we examine $\tilde{b}(n, d)$ for $d=O(\log n)$ by defining $\omega$ from

$$
d=\omega \log n, \quad 0<\omega<\frac{1}{\log (1 / p)}
$$

and then set

$$
\tilde{b}(n, d)=\log ^{2}(n) \mathcal{F}(\omega)
$$


Then we approximate (14) again by $\tilde{b}(n, d) \sim \tilde{b}(n p, d-1)$ which in view of $(79)$ becomes

$$
\begin{aligned}
\log ^{2}(n) \mathcal{F}(\omega) & \sim \log ^{2}(n p) \mathcal{F}\left(\frac{d-1}{\log (n p)}\right) \\
& \sim(\log n+\log p)^{2} \mathcal{F}\left(\omega-\frac{1}{\log n}-\frac{\omega \log p}{\log n}+O\left(\log ^{-2} n\right)\right) .
\end{aligned}
$$

From (80) we obtain the following limiting ODE:

$$
0=-\mathcal{F}^{\prime}(\omega)(1+\omega \log p)+2 \log p \mathcal{F}(\omega) .
$$

The solution to $(81)$ is

$$
\mathcal{F}(\omega)=(1+\omega \log p)^{2} \mathcal{F}_{*}
$$

where $\mathcal{F}_{*}$ is a constant. For $\omega \rightarrow 0$, the expansion in $(79)$ behaves as $\mathcal{F}_{*} \log ^{2}(n)$ and if we match the $\omega$-scale result to the $d=O(1)$ result in Theorem 1 , we conclude that

$$
\mathcal{F}_{*}=\frac{1}{2 h} \frac{1}{\log (1 / p)} \text {. }
$$

Finally, by asymptotically matching (79) as $\omega \rightarrow[\log (1 / p)]^{-1}$ to the approximation in (73) and (76), for $\xi \rightarrow-\infty$, we conclude that

$$
\mathcal{B}_{0}(\xi) \sim \frac{1}{2 h} \log ^{2}(p) \xi^{2}, \quad \xi \rightarrow-\infty .
$$

Note that $\xi$ and $\omega$ are related by

$$
1+\omega \log p=\frac{\log p}{\log n} \xi
$$

so that when $0<\omega<[\log (1 / p)]^{-1}$ we have $\xi<0$.

To summarize the formal results in this subsection, our analysis suggests that the asymptotics of $\tilde{b}(n, d)$ are different for the three cases:

(i) $n=O(1), d \rightarrow \infty$ (where (31) holds),

(ii) $\xi=d-\log _{1 / p}(n)=O(1)$ where $(76)$ holds, and

(iii) $d=O(\log n)$ where $\tilde{b}(n, d) \sim(2 h)^{-1}(1+\omega \log p)^{2} \log ^{2} n /(-\log p)$ with $d=\omega \log n$ and $0<\omega<[\log (1 / p)]^{-1}$.

The result in Theorem 1 appears to be a limiting case of the $d=O(\log n)$ expansion, when it is expanded for $\omega \rightarrow 0$. However, Theorem 1 also gives the second term $(O(\log n))$ in the asymptotic series for $d=O(1)$.

We have only given the asymptotic behaviors of $\mathcal{B}_{0}(\xi)$ as $\xi \rightarrow \pm \infty$. To get a more explicit expression for $\tilde{b}(n, d) \sim \mathcal{B}(n, \xi)$ in (76) we again argue that $\tilde{b}(n, d) \sim b_{*}(n, d)$ 
holds for $\xi=O(1)$ (in fact this relation fails only for $n=O(1)$ and $d \rightarrow \infty$ ). If instead of defining $\xi$ from (72) we let

$$
d=\left\lfloor\log _{1 / p}(n)\right\rfloor+\xi^{\prime}=\log _{1 / p}(n)+\xi^{\prime}-\left\{\log _{1 / p}(n)\right\},
$$

where $\{\cdot\}$ denotes the fractional part, then

$$
p^{d} n=p^{\xi^{\prime}} \exp \left[-d \log (1 / p)\left\{\log _{1 / p}(n)\right\}\right]
$$

and for $n \rightarrow \infty$ with $\xi, \xi^{\prime}=O(1)$ the limiting form of $(68)$ is

$$
\frac{-1}{2 \pi i} \int_{B r} p^{-s \xi^{\prime}} \mathcal{M}(s) p^{s d\left\{\log _{1 / p}(n)\right\}} d s
$$

with $\mathcal{M}(\cdot)$ as in (67). We therefore conjecture that the right side of (76) is given explicitly by $(84)$, with $\xi$ in $(76)$ replaced by $\xi^{\prime}$ in $(83)$.

\section{References}

[1] Y. Choi and W. Szpankowski, Compression of Graphical Structures: Fundamental Limits, Algorithms, and Experiments, IEEE Transaction on Information Theory, 58, 620-638, 2012.

[2] L. Devroye, A Study of Trie-Like Structures Under the Density Model, Annals of Applied Probability, 2, 402-434, 1992.

[3] M. Drmota, Random Trees, Springer, New York, 2009.

[4] M. Drmota and W. Szpankowski, The Expected Profile of Digital Search Trees, J. Combin. Theory, Ser. A, 118, 1939-1965, 2011.

[5] P. Flajolet, X. Gourdon, and P. Dumas, Mellin Transforms and Asymptotics: Harmonic Sums, Theoretical Computer Science, 144, 3-58, 1995.

[6] P. Flajolet, Singularity Analysis and Asymptotics of Bernoulli Sums, Theoretical Computer Science, 215, 371-381, 1999.

[7] P. Flajolet and R. Sedgewick, Analytic Combinatorics, Cambridge University Press, Cambridge, 2009.

[8] P. Jacquet, and W. Szpankowski, Analytical Depoissonization and its Applications, Theoretical Computer Science, 201, 1-62, 1998.

[9] P. Jacquet and W. Szpankowski, Entropy Computations via Analytic Depoissonization, IEEE Trans. Information Theory, 45, 1072-1081, 1999.

[10] S. Janson and W. Szpankowski, Analysis of an Asymmetric leader Election Algorithm, Electronic J. of Combinatorics, 4, R17, 1997.

[11] C. Knessl, and W. Szpankowski, Asymptotic Behavior of the Height in a Digital Search Tree and the Longest Phrase of the Lempel-Ziv Scheme, SIAM J. Computing, 30, 923-964, 2000. 
[12] D. Knuth, The Art of Computer Programming. Sorting and Searching, Vol. 3, Second Edition, Addison-Wesley, Reading, MA, 1998.

[13] G. Louchard, Exact and Asymptotic Distributions in Digital and Binary Search Trees, RAIRO Theoretical Inform. Applications, 21, 479-495, 1987.

[14] H. Mahmoud, Evolution of Random Search Trees, John Wiley \& Sons Inc., New York, 1992.

[15] G. Park, H.K. Hwang, P. Nicodème, and W. Szpankowski, Profile of Tries, SIAM J. Computing, 8, 1821-1880, 2009.

[16] B. Pittel, Asymptotic Growth of a Class of Random Trees, Annals of Probability, 18, 414-427, 1985.

[17] B. Pittel, Path in a Random Digital Tree: Limiting Distributions, Advances in Applied Probability, 18, 139-155, 1986.

[18] W. Szpankowski, A Characterization of Digital Search Trees from the Successful Search Viewpoint, Theoretical Computer Science, 85, 117-134, 1991.

[19] W. Szpankowski, Average Case Analysis of Algorithms on Sequences, Wiley, New York, 2001. 